\title{
Ueber Isocinchonin;
}

\author{
von 0 . Hesse.
}

(Eingelaufen den 6. Juni 1890.)

In einer früberen Mittheilung *) habe ich die neuen Isomeren einiger Chinaalkaloide, welche aus letzteren mittelst Schwefelsäure erhältlich sind, nur kurz beschrieben, indem ich hoffte, den ausführlichen Bericht über die gesammte Untersuchung bald folgen lassen zu kōnnen. Besondere Umstände bestimmen mich jedoch jetzt, diese Untersuchung in mehreren Theilen zu behandeln; daher erlaube ich mir, mit der Mittheilung über das Isocinchonin zu beginnen. Da inzwischen über dieses Alkaloïd auch von Anderen **) berichtet wurde, so möchte ich jedoch die Bemerkung beifügen, dafs meine Untersuchung desselben beim Erscheinen der citirten Mittheilung in diesen Annalen in der Hauptsache schon beendet war und dafs jene Publicationen, auf die ich weiter unten zurückkommen werde, einige Controlversuche bedingten, welche zum Theil allerdings erst in jüngster Zeit ausgeführt werden konnten.

Wird käufliches schwefelsaures Cinchonin oder auch die Base selbst in dem Verhältnifs von $1: 5$ bis 10 in concentrirter Schwefelsäure gelöst, wobei sich die Masse etwas 'erwärmt, so erhält man zunächst eine Auflösung von Cinchonin, aus welcher das Alkaloï, mit allen seinen Eigenschaften begabt, wieder erhältlich ist, wenn man die Lösung sofort oder wenige Minuten später mit Wasser verdünnt und nun mit Natronlauge niederschlägt. Lärst man dagegen diese Lōsung

*) Diese Annalen 248, 147.

**) Comstock und Koenigs, Ber. d. deutsch. chem. Ges. 20, 510 ; Jungflei s $\mathrm{h}$ und Léger, Moniteur scientifique [4] $\curvearrowright$, 188; Caventou und Girard, daselbst [4] $\Xi, 217$. 
länger als einige Minuten stehen, so verwandelt sich das Cinchonin mehr und mehr in Isocinchonin. Erwärmen der Lösung beschleunigt diese Umwandlung, ist aber dazu nicht erforderlich. Wendet man 2. B. $30 \mathrm{~g}$ Sulfat und $150 \mathrm{~g}$ Säure an, so ist diese Veränderung bei gewöhnlicher Temperatur nach 24 Stunden, beim Erwärmen auf 60 bis $80^{\circ}$ nach 6 Stunden beendet. Bei Anwendung einer relativ gröfseren Menge Säure, etwa der zehnfachen von der des Cinchonins, vollzieht sich diese Umwandlung ebenfalls rascher, auch ist die Dauer dieses Processes von der absoluten Menge Cinchonin, welche angewandt wurde, abhängig. Ich erwähne deshalb, dafs ich zu meinen Versuchen 10 bis $30 \mathrm{~g}$ Sulfat (oder eine äquivalente Menge Cinchonin) anwandte und die eventuell wieder erkaltete Lösung erst nach 24 Stunden, wie das gerade pafste, weiter verarbeitete, da Vorversuche ergeben hatten, dafs selbst eine mehrtägige Aufbewahrung der Lōsung bei gewōhnlicher Temperatur auf das Resultat keinen Einflufs ausübt.

Die so vorbereitete saure Lösung wurde alsdann in kaltes Wasser eingetragen und mit Natronlauge nahezu neutralisirt, wobei sie sich stark erwärmte, dann nach dem vollständigen Erkalten mit Natronlauge übersättigt und mit vielem Aether ausgeschüttelt. Von der ätherischen Lösung wurde hierauf ein gröfserer Theil Aether abdestillirt und die rückständige Flüssigkeit sich nun selbst überlassen, wobei sich eine aus kleinen Nadeln bestehende Krystallisation (A) abschied, von welcher weiter unten die Rede sein wird. Sobald eine Zunahme dieser Krystallisation nicht mehr wahrnehmbar war, wurde die Lösung abgegossen und weiter verdunstet, wobei ein öliger Rückstand blieb, der alsbald strahlig krystallinisch erstarrte. Die Krystallmasse wurde dann abgeprefst und durch wiederholtes Umlösen aus wenig Aether gereinigt. Diese Reinigung kann man auch in der Art bewirken, dafs man den aus der ätheri- 
schen Lösung erhaltenen Rückstand in der Wärme mit verdünnter Salzsäure neutralisirt, worauf beim Erkalten salzsaures Isocinchonin krystallisirt, welches schon nach einmaligem Krystallisiren aus heifsem Wasser rein erhalten wird; aus diesem Salze wird dann die Base init Natronlauge und Aether abgeschieden, welch' letaterer es nun beim langsamen Verdunsten in schönen Prismen abscheidet, die, wie die Analyse besagt, nach der Formel $\mathrm{C}_{19} \mathrm{H}_{22} \mathrm{~N}_{2} \mathrm{O}$ zusammengesetzt sind.

$0,2658 \mathrm{~g}$ bei $100^{\circ}$ getrockneter Substanz gaben $0,754 \mathrm{CO}_{2}$ and $0,1785 \mathrm{H}_{2} \mathrm{O}$.

$\begin{array}{ccc} & \text { Berechnet } & \text { Gefunden } \\ \text { C } & \mathbf{7 7 , 5 5} & 77,36 \\ \text { H } & \mathbf{7 , 4 8} & 7,46 .\end{array}$

Das Isocinchonin krystallisirt aus reinem Aether in derben farblosen, glasglänzenden Prismen, die wasserfrei sind und (im Roth'schen Apparate) bei $125^{\circ}$ schmelzen. In höherer Temperatur verflüchtigt es sich unverändert und destillirt namentlich im Wasserstoffstrom ziemlich leicht. Es löst sich leicht in Alkohol, Aether, Aceton, Benzin, Chloroform, nicht in Wasser oder Alkalien, bläut in alkoholischer Lösung rothes Lackmuspapier, läfst dagegen Phenolphtaleïnpapier unverändert. Es ist in seinen Lösungen optisch activ; jedoch dreht es im Gegensatz zu Cinchonin die Ebene des polarisirten Lichtes nach links und wirkt in concentrirter Lösung relativ stärker als wie in verdünnter. So beträgt z. B. für die Lösung in absolutem Alkohol bei $\mathrm{t}=15^{\circ}$ und $\mathrm{p}=1(\alpha)_{\mathrm{D}}=-53,7^{\circ}$, bei $\mathrm{p}=3(\alpha)_{\mathrm{D}}=-55,6^{\circ}$.

In verdünnten Säuren löst sich das Isocinchonin leicht; diese Lösungen, welche hitter schmecken und keine Fluorescenz zeigen, geben mit Natronlauge sowohl, wie mit Ammoniak harzige Fäliungen, etwas löslich in letzterem. Es bildet mit den Säuren neutrale und einfach saure Salze, welche meist recht gut krystallisiren und von denen die folgenden näher untersucht wurden. 
Neutrales salzsaures Isocinchonin wird wie oben angegeben erhalten. Dasselbe krystallisirt in sechsseitigen glasglänzenden Prismen, welche, sofern sie gruppirt sind, auf der freien Seite durch ein Doma, wenn isolirt, beiderseits durch Domen abgestumpft sind. Es löst sich leicht in heifsem Wasser, ziemlich leicht in Alkohol und in Chloroform, wenig in kaltem Wasser, nicht in Aether und kalter Kochsalzlösung. Es enthält 1 Mol. Krystallwasser, das es weder im Exsiccator, noch bei $100^{\circ}$ verliert. Erst gegen $115^{\circ}$ beginnt die Entwickelung von Wasser, die sich beim längeren Erhitzen auf diese Temperatur auf etwa $1 / 3$ Mol. erstreckt, während der Rest erst bei 140 bis $150^{\circ}$, wenngleich äufserst schwierig, entweicht. Vollkommen entwässert, schmilat es dann bei $201^{\circ}$.

$0,3018 \mathrm{~g}$ luftrockene Substanz gaben $0,725 \mathrm{CO}_{2}$ und $0,1835 \mathrm{H}_{8} \mathrm{O}$. $0,3065 \mathrm{~g}$

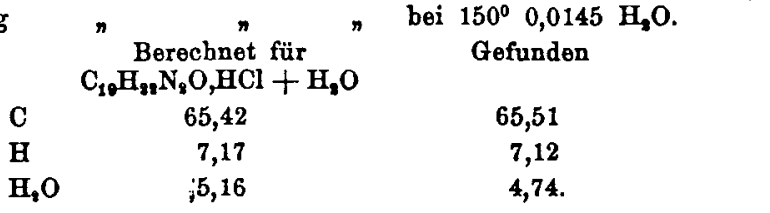

Das salzsaure Isocinchonin gab ferner bei der polarimetrischen Prüfung bei $t=15^{\circ}$ in wässeriger Lösung und $p$ $=1(\alpha)_{\mathrm{D}}=-68,6^{\circ}, \mathrm{p}=2(\alpha)_{\mathrm{D}}=-71,2^{\circ}, \mathrm{p}=2$ in Wasser und $2 \mathrm{Mol}$. $\mathrm{HCl}(\alpha)_{\mathrm{D}}=-28,3^{\circ}$, desgleichen in Wasser, dagegen $5 \mathrm{Mol}$. $\mathrm{HCl}(\alpha)_{\mathrm{D}}=-27,9^{\circ}, \mathrm{p}=5$ in Wasser und $2 \mathrm{Mol}$. $\mathrm{HCl}(\alpha)_{\mathrm{D}}=-34,0^{n}$, ferner bei $\mathrm{p}=2$ in Chloroform und $\mathrm{t}=22^{\circ}(\alpha)_{\mathbf{D}}=-149^{\prime \prime}$.

Neutrales Chloroplatinat. - Chlorplatinnatrium giebt in der wässerigen Auflösung des salzsauren Salzes einen blafsgelben, flockigen, in kaltem Wasser schwer lõslichen Niederschlag.

$0,1800 \mathrm{~g}$ lufttrockener Substanz gaben bei $130^{\circ} 0,0095 \mathrm{H}, \mathrm{O}$ und dann beim Verbrennen $0,0335 \mathrm{Pt}$.

Berechnet für

Gefunden

$\begin{array}{ccr}\left(\mathrm{C}_{10} \mathrm{H}_{12} \mathrm{~N}_{2} \mathrm{O}\right)_{8}, \mathrm{PtCl}_{6} \mathrm{H}_{2}+3 \mathrm{H}_{4} \mathrm{O} & \\ \mathrm{Pt} & 18,53 & 18,61 \\ 3 \mathrm{H}_{2} \mathrm{O} & 5,13 & 5,27 .\end{array}$


Saures Chloroplatinat. - Platinchlorid erzeugt in der wässerigen Lösung des Chlorhydrats einen gelben amorphen flockigen Niederschlag (A), welcher sich ziemlich rasch, namentlich beim Erwärmen der Lösung, in orangefarbene Nadeln (B) umsetzt. Das Salz löst sich ziemlich schwer in Salzsäure, kaum in kaltem Wasser.

A. $0,284 \mathrm{~g}$ lufttrackener Substanz gaben bei $120^{\circ} 0,0137 \quad \mathrm{H}_{\mathbf{q}} \mathrm{O}$ und beim Verbrennen 0,0757 Pt.

B. $0,6475 \mathrm{~g}$ lufttrockener Substanz gaben bei $120^{\circ} 0,0175 \mathrm{H}_{\mathbf{2}} \mathrm{O}$ und beim Verbrennen 0,1752 Pt.

\begin{tabular}{|c|c|c|}
\hline \multicolumn{2}{|c|}{$\begin{array}{c}\text { Berechnet für } \\
\mathrm{C}_{19} \mathrm{H}_{2 n} \mathrm{~N}_{2} \mathrm{O}, \mathrm{PtCl}_{6} \mathrm{~B}_{2}+2 \mathrm{H}_{2} \mathrm{O}\end{array}$} & $\begin{array}{c}\text { Gefunden } \\
\text { A. }\end{array}$ \\
\hline $\mathbf{P t}$ & 26,32 & 26,65 \\
\hline $2 \mathrm{H}_{3} \mathrm{O}$ & 4,86 & 4,85 \\
\hline \multicolumn{2}{|c|}{$\begin{array}{c}\text { Berechnet für } \\
\mathrm{C}_{19} \mathrm{H}_{82} \mathrm{~N}_{8} \mathrm{O}, \mathrm{PtCl}_{6} \mathrm{H}_{2}+\mathrm{H}_{2} \mathrm{O}\end{array}$} & $\begin{array}{l}\text { Gefunden } \\
\text { B. }\end{array}$ \\
\hline $\mathbf{P t}$ & 26,96 & 27,05 \\
\hline $\mathrm{H}_{8} \mathrm{O}$ & 2,46 & 2,70 \\
\hline
\end{tabular}

Saures Chlorgolddoppelsalz. - Beim Vermischen der wässerigen Lösung des salzsauren Salzes mit Goldchloridlösung entsteht ein schön gelber, flockiger, amorpher Niederschlag, schwer löslich in kaltem Wasser und nach dem Trocknen bei $100^{\circ}$ der Formel nach $\mathrm{C}_{19} \mathrm{H}_{82} \mathrm{~N}_{2} \mathrm{O}, 2 \mathrm{AuCl}_{4} \mathrm{H}$ zusammengesetzt.

$0,4273 \mathrm{~g}$ bei $100^{\circ}$ getrockneter Substanz gaben 0,2718 Au.

$$
\begin{array}{ccc} 
& \text { Berechnet } & \text { Gefunden } \\
\text { Au } & 40,45 & 40,23 .
\end{array}
$$

Quecksilberchloridsalz. - Beim Vermischen der heifsen wässerigen Lösung des Chlorhydrats mit einer erwärmten Lōsung von Quecksilberchlorid erfolgt milchige Trübung derselben und bald die Abscheidung der Quecksilberverbindung in kleinen concentrisch gruppirten Nadeln, welche kein Krystallwasser enthalten.

$0,1195 \mathrm{~g}$ bei $100^{\circ}$ getrockneter Substanz gaben $0,0840 \mathrm{AgCl}$.

$$
\begin{array}{ccc} 
& \begin{array}{c}
\text { Berechnet für } \\
\mathrm{Cl}
\end{array} & \text { Gefunden } \\
\mathrm{C}_{19} \mathrm{H}_{\mathbf{2 8}} \mathrm{N}_{8} \mathrm{O}, \mathrm{HCI}+\mathrm{HgCl}_{2} & 17,70 & 17,39 .
\end{array}
$$

Annalen der Chemio 260. Bd. 
Erfolgt die Fällung in angesäuerter Lösung, so entsteht ein wechselndes Gemisch von dieser Verbindung und $\mathrm{C}_{19} \mathrm{H}_{28} \mathrm{~N}_{2} \mathrm{O}$, $2 \mathrm{HCl}+\mathrm{HgCl}_{8}$.

Zinkchloridverbindung. - Wird die heifse wässerige, schwach angesäuerte Lösung des Chlorhydrats mit Zinkchloridlösung vermischt, so erfolgt sofort die Abscheidung der Doppelverbindung in kleinen farblosen Nadeln, welche wasserfrei sind, bei $260^{\circ}$ schmelzen, sich schwer in Zinkchloridlosung, gut in heifsem Wasser lösen.

$0,340 \mathrm{~g}$ bei $100^{\circ}$ getrockneter Substanz gaben 0,1975 Isocinchonin.

$$
\begin{aligned}
& \text { Berechnet für } \\
& \mathrm{C}_{19} \mathrm{H}_{29} \mathrm{~N}_{8} \mathrm{O} \mathrm{C}_{19} \mathrm{H}_{\mathbf{2 8}} \mathrm{N}_{8} \mathrm{O}, 2 \mathrm{HCl}+\mathrm{ZnCl}_{\mathbf{8}}
\end{aligned}
$$

Neutrales jodwasserstoffsaures 1socinchonin, durch Wechselzersetzung von salzsaurem Isocinchonin mit Jodkalium in heifser wässeriger Lösung erhalten, krystallisirt in weifsen zarten Nadeln, welche sich ziemlich leicht in heifsem Wasser, schwer in kaltern Wasser, nicht in überschüssiger Jodkaliumsolution lösen.

$0,3855 \mathrm{~g}$ lufttrockener Substanz gaben bei $130^{\circ} \quad 0,0150 \quad \mathrm{H}_{\mathbf{2}} \mathrm{O}$.

$$
\begin{array}{ccc} 
& \begin{array}{c}
\text { Berechnet für } \\
\mathrm{H}_{\mathbf{2}} \mathrm{O}
\end{array} & \text { Gefunden } \\
\mathrm{H}_{\mathbf{8 2}} \mathrm{N}_{\mathbf{2}} \mathrm{O}, \mathrm{HJ}+\mathrm{H}_{\mathbf{2}} \mathrm{O} & \\
4,09 & 3,87 .
\end{array}
$$

Neutrales sulfocyanwasserstoffsaures Isocinchonin, in analoger Art wie das vorgenannte Salz erhalten, krystallisirt in zarten, platten Nadeln, die sich in heifsem Wasser sehr leicht lösen, in kaltem Wasser aber so schwer, dafs Natronlauge oder Ammoniak in solcher Lösung keine Trübung, geschweige denn einen Niederschlag erzeugen. Es enthält kein Krystallwasser und ist daher nach $\mathrm{C}_{19} \mathrm{H}_{29} \mathrm{~N}_{2} \mathrm{O}$, CNSH zusammengesetzt.

Neutrales schwefelsaures Isocinchonin, durch Sättigen der Base mit verdünnter Schwefelsäure zu erhalten, krystallisirt in langen rhombischen, glatt abgestumpften Prismen, welche sich leicht in Alkohol und Wasser lösen. 
0,3645 g luftrockener Substanz gaben bei $120^{\circ} 0,0505 \mathrm{H}_{9} \mathrm{O}$, sowio $0,1065 \mathrm{SO}, \mathrm{Ba}$.
Berechnet für $\left(\mathrm{C}_{10} \mathrm{H}_{22} \mathrm{~N}_{2} \mathrm{O}\right)_{2}, \mathrm{SO}_{4} \mathrm{H}_{2}+6 \mathrm{H}_{8} \mathrm{O}$
$\mathrm{SO}_{\mathrm{s}}$ 10,07
Gefunden
$6 \mathrm{H}_{2} \mathrm{O}$
13,60
10,09
13,85 .

Saures Sulfat. - Wird die vorstehende Verbindung noch mit 1 Mol. Schwefelsāure zusammengebracht, so krystallisirt aus der māfsig concentrirten wässerigen Lōsung das saure Sulfat in sehr langen zarten Nadeln, die sich leicht in heifsem Wasser, mäfsig in kaltem, lösen.

$0,2455 \mathrm{~g}$ lufttrockener Substanz gaben bei $100^{\circ} 0,0388 \mathrm{H}, \mathrm{O}$, sowie $0,1195 \mathrm{SO}_{4} \mathrm{Ba}$.

$\begin{array}{rcr} & \begin{array}{c}\text { Berechnet für } \\ \mathrm{SO}_{8}\end{array} & \text { Gefunden } \\ 4 \mathrm{C}_{2} \mathrm{O} & 17,24 & 17,42 \\ \mathrm{~N}_{2} \mathrm{O}, \mathrm{SO}_{4} \mathrm{H}_{2}+4 \mathrm{H}_{2} \mathrm{O} & \\ & 15,52 & 16,34 .\end{array}$

Neutrales oxalsaures 1socinchonin, durch Sättigen der Base mit Oxalsãure in wässeriger Lõsung erhalten, bildet farblose derbe Nadeln, welche sich leicht in Wasser, nicht in Aether lösen.

0,426 g lufttrockener Substanz gaben bei $100^{\circ} 0,031 \mathrm{H}_{3} \mathrm{O}$ und nach dem Verbrennen des oxalsauren Calciums u. s. w. 0,0795 $\mathrm{SO}_{4} \mathrm{Ca}$.

$\begin{array}{ccc} & \begin{array}{c}\text { Berechnet für } \\ \left(\mathrm{C}_{19} \mathrm{H}_{29} \mathrm{~N}_{2} \mathrm{O}\right)_{2}, \mathrm{C}_{2} \mathrm{O}_{4} \mathrm{H}_{2}\end{array} & \text { Gefunden } \\ \mathrm{C}_{2} \mathrm{O}_{4} \mathrm{H}_{2} & 12,29 & 12,40 \\ 3 \mathrm{H}_{2} \mathrm{O} & 7,37 & 7,35 .\end{array}$

Soweit die Salze des Isocinchonins. Es lag nahe zu versuchen, das Isocinchonin in Cinchonicin überzuführen resp. das letztere in Isocinchonin. In erster Beziehung hatte ich früher gezeigt, dafs das Cinchonin und Cinchonidin, die genuinen Isomeren unseres Alkaloïds, alsbald in Cinchonicin übergehen, wenn deren einfachschwefelsaure Salze einer Temperatur von etwa $135^{\circ}$ ausgesetzt werden. Wird mit dem sauren Isocinchoninsulfat genau so verfahren, so färbt sich dasselbe zunächst gelblich und beginnt dann zu schmelzen, allein es entsteht weder bei dieser Temperatur, noch bei 
einer um 10 bis $15^{\circ}$ höher gelegenen Cinchonicin; mit Ausnahme eines geringen Abganges für färbende Substanz bleibt das Alkaloïd unverändert. Desgleichen verändert es sich nicht, wenn sein Oxalat bei gewöhnlicher Temperatur in concentrirter Schwefelsäure gelöst oder diese Lösung stundenlang auf 120 bis $130^{\circ}$ erhitzt wird.

Löst man dagegen oxalsaures Cinchonicin in concentrirter Schwefelsäure, so resultirt eine stark gefärbte loösung, die mehr und mehr dunkler wird, namentlich beim Erwärmen. Die Untersuchung dieser Lösung ergab, dafs sich das Cinchonicin in eine dunkelgefärbte, in Aether lösliche Base verwandelt halte, welche gleich dem Cinchonicin amorph ist, jedoch nicht die Fähigkeit besitzt, krystallisirbare Salze zu bilden.

Hieraus folgt nun, dafs unter den bekannten Bedingungen eine Umwandlung des Isocinchonins in Cinchonicin oder umgekehrt nicht stattindet und überhaupt eine solche Umwandlung sehr unwahrscheinlich ist.

Was ferner die obengenannte Krystallisation A betriff, so habe ich dieselbe stets bei Anwendung von kāuflichem neutralen Sulfat erhalten, wenngleich in sehr geringer Menge. Diese Krystallisation besteht nun im wesentlichen aus Hydrocinchonin und Apocinchonin; beide lassen sich zusammen daraus abscheiden, wenn man die Masse in concentrirter Schwefelsäure löst und einige Stunden auf 80 bis $100^{\circ}$ erwärmt oder wenn man dieselbe in verdünnter Schwefelsäure auflöst und dazu tropfenweise eine verdünnte Lösung von Kaliumpermanganat bis zur bleibenden Rothfärbung bringt. Werden dann die frisch gefältten Alkaloỉde mit Aether behandeit, so wird hauptsächlich das Apocinchonin gelöst, während der gröfsere Theil des Hydrocinchonins ungelöst bleibh, so das es nun in Form des neutralen Sulfats ohne Schwierigkeit völlig rein erhalten werden kann. 
Dafs das käufliche Cinchoninsulfat stets Hydrocinchonin enthalte, wurde schon von Willm und $C$ aventou*), den Entdeckern dieser Base, vermuthet, aber es ist bis jetzt Niemandem gelungen, dasselbe ohne Anwendung von Kaliumpermanganat aus dem Cinchonin abzuscheiden, wie vorstehend geschehen. Aber selbst wenn das Cinchonin ganz frei von Hydrocinchonin ist, wie z. B. sehr leicht der Fall sein kann bei dem Cinchonindisulfat, so erhält man doch eine allerdings sehr geringe Krystallisation, wenn man dásselbe in concentrirter Schwefelsäure auflöst und diese Lösung 24 Stunden sich selbst überlärst; nur besteht diese Krystallisation im wesentlichen aus Apacinchonin. Letzteres bildet sich bei diesem Procefs immer etwas; es geht nicht in Isocinchonin über und ist überhaupt in concentrirter Schwefelsäure äufserst beständig; so dafs wir es schliefslich in fraglicher Krystallisation antreffen können.

Nach Mittheilung dieser Versuche erübrigt nur noch, die oben erwähnten bezüglichen Untersuchungen von $\mathrm{Coms}$ t ock und Koenigs, Jungfleisch und Léger, sowie von Caventou und Girard einer Erörterung zu unterwerfen. Comstock und Koenig $s^{*}$ ) erhielten das Isocinchonin,

*) Diese Annalen Suppl. 2, 247.

**) Ber. d. deutsch. chem. Ges. 20, 2521. Diese Mittheilung lief am 13. Aug. 1887 ein und erschien am 13. Sept. dess. Jahres. Ich schrieb damals sofort an Herrn Dr. Koonigs, dafs ich einige Monate früher eine Mittheilung über das gleiche Alkaloïd an die Red. der Annalen eingesandt und mir die weitere Untersuchung des Gegenstandes vorbehalten hätte. In der hierauf stattfindenden Zusammenkunft (Ende Sept. 1887) verstandigten wir uns in dieser Sacho dahin, dafs ich mich auf die Untersuchung der Salze, die ziemlich weit vorgeschritten war, beschränken würde. Als dann später Jung fleisch und Léger über ihre Untersuchung über das Verhalten des Cinchonins zu Schwefelsalure der Pariser Akademie berichteten, stellte ich meine Untersuchung ein, bis jene von Jungfleisch und Léger in 
das sie unter demselben Namen beschrieben wie ich, durch 10 bis 12 stūndiges Kochen von $10 \mathrm{~g}$ zweifach bromwasserstoffsaurem Hydrobromcinchonins mit $20 \mathrm{~g}$ Kali und $300 \mathrm{cbcm}$ absolutem Alkohol neben etwas Cinchonin. Eine Wiederholung dieses Versuchs habe ich nicht für nöthig gehalten, da meine Beobachtungen mit den Angaben von Comstock und Königs, soweit dieselben vergleichbar waren, vollkommen übereinstimmten, mit Ausnahme des Schmelzpunktes, den Co mstock und Königs zu 125 bis $127^{\circ}$ angaben, während ich denselben scharf bei $125^{\circ}$ fand. Dieser Differenz glaubte ich indefs keine Bedeutung beilegen zu sollen. Auch aus Hydrochlorcinchonin wollen diese Chemiker nach dem gleichen Verfahren Isocinchonin erhalten haben, das sie jedoch nicht näher untersuchten, sodafs nicht ausgeschlossen ist, dafs das in dieser Art erhaltene Alkaloĩd mit dem unter den gleichen Verhältnissen aus Hydrochlorapocinchonin entstehenden, in Aether leicht löslichen, aber vom Isocinchonin bestimmt verschiedenen Alkaloï identisch ist. Ueber diesen Punkt hoffe ich in nicht zu ferner Zeit näheres berichten zu kōnnen.

Was die erste Mittheilung von Jung fleis $\mathrm{ch}$ und Léger über fraglichen Gegenstand betriff, so datirt dieselbe vom 19. December 1887. Sie erwähnen in derselben nicht jene von Comstock und $K o ̈ n i g s$, welche einige Monate früher erschien, obgleich nahegelegt war, dafs ihr Cinchonicin mit dem schon bekannten Isocinchonin identisch sei. Jung $\mathrm{fle}$ is $\mathrm{ch}$ und $\mathrm{L}$ é g er erhitzten in ihrer Untersuchung eine Auflösung von angeblich reinem schwefelsauren Cinchonin in dem vierfachen Gewicht eines Gemisches von Schwefelsäure $(d=1,84)$ und W asser zu gleichen Theilen bei $120^{\circ} 48$ Stunden lang am Rückflufskühler und erhielten so nicht weniger als sechs Basen, wovon

Aussicht gestellte ausführliche Mittheilung über Cinchonigin erschien. Ich glaube dies anführen zu sollen, um jederlei Mifsdeutungen darüber vorzubeugen. 
vier isomer mit Cinchonin waren und die sie, dem Alphabet folgend, Cinchonibin, Cinchonifin, Cinchonigin und Cinchonilin nannten, während die beiden andern Oxydationsproducte des Cinchonins sein sollten, welche sie mit $\alpha$ - und $\beta$-0xycinchonin bezeichneten. Die Entstehung der letzteren Basen wird damit erklärt, dafs während dieses Processes sich Verbindungen der Schwefelsäure mit Cinchonin bilden sollen, welche dann durch das zurückfliefsende Wasser oxydirt würden. Gegen diese Erklärung würde jedoch vorzubringen sein, dafs dieses Wasser wohl etwa gebildete Sulfoverbindungen verseifen und somit in Schwefelsäure und Cinchonin oder eventuell dessen Umlagerungsproducte zerlegen, keineswegs aber oxydiren kônnte, ganz abgesehen davon, dafs sich unter den obwaltenden Verhältnissen überhaupt keine Schwefelsäurederivate des fraglichen Alkaloïds bilden.

Wie nicht anders zu erwarten, habe ich denn auch bei Wiederholung dieses Versuches keine Oxybasen erhalten. Dabei ging ich vom Cinchoninbisulfat aus, um nicht den Complicationen zu begegnen, die das käufliche „reine" schwefelsaure Cinchonin durch einen Gehalt an Hydrocinchonin zur Folge hat. Bei Anwendung dieses Präparates habe ich aber auch kein Cinchonibin erhalten, sondern neben kleinen Mengen von Apocinchonin nur Cinchonigin. Da nun aber das ${ }^{\text {reine }}$ " schwefelsaure Cinchonin des Handels auch Hydrocinchonin enthält, das unfehlbar bei der Vorschrift von Jungfleisch und $\mathrm{L} \dot{\mathrm{eg}} \mathrm{er}$ wieder erhalten wird, wäbrend diese Chemiker es nicht erwähnen, so schliefse ich aus allem, dafs das Hydrocinchonin sowohl, wie das aus dem Cinchonin entstandene Apocinchonin in dem Cinchonifin und Cinchonilin ein Unterkommen fanden.

Was nun das Cinchonigin anbelangt, so ist dasselbe nichts anderes als das ron mir zuerst rein dargestellte Isocinchonin. Die Angaben von Jung fle is ch und Léger über „Cincho- 
nigin" stimmen zwar nicht ganz mit meinen bezüglichen $\mathrm{Be}-$ obachtungen überein, allein meine Vergleichung des nach dem Verfahren von $J$ ungfleisch und Léger dargestellten Alkaloïds mit lsocinchonin ergab vollkommene Ueboreinstimmung. Es gelang mir daher auch nicht, aus jenem Material ein neutrales Oxalat darzustellen, das wie die genannten Chemiker angeben, in Tafeln krystallisiren und nach $\left(\mathrm{C}_{19} \mathrm{H}_{82} \mathrm{~N}_{2} \mathrm{O}\right)_{2}, \mathrm{C}_{2} \mathrm{H}_{2} \mathrm{O}_{4}$ $+5 \mathrm{H}_{2} \mathrm{O}$ zusammengesetzt sein soll.

Nicht viel anders als wie in dem eben angeführten Prozers verläuft die Reaction bei dem von $\mathrm{Caventou}$ und Girard angegebenen Verfahren, worüber sie der Pariser Akademie in der übernáchstfolgenden Sitzung (2. Jan. 1888) berichteten. In der Absicht, an das Molekül des Cinchonins die Gruppe $\mathrm{CO}$ anzufügen, unterwarfen $\mathrm{Caventou}$ und Girard das fragliche Alkaloïd in der Auflösung von Schwefelsäuremonohydrat einer mebrstündigen Einwirkung von Oxalsäure bei einer Temperatur von 125 bis $130^{\circ}$, jedoch, wie zu erwarten, ohne den erwünschten Erfolg. Dagegen war es ihnen möglich, aus der nun braunen Lösung aufser einer in Aether unlöslichen Parthie, die sie glauben in der Hauptsache für unverändertes Cinchonin ansprechen zu sollen, noch ein in Aether und Benzin lösliches und daraus hrystallisirbares Alkaloïd abzuscheiden, sowie eine in Aether lösliche, in Benzin aber unlösliche basische Substanz. Jenes aus Aether und Benzin krystallisirbare Alkaloïd besafs derartige Eigenschaften, dafs die Identität desselben mit dem Cinchonigin von $\mathrm{Jung} f \mathrm{le}$ is c h und $L$ ég er als sicher angenommen werden konnte.

Bei der Wiederholung dieses Versuchs, bei welchem ich anstatt vom Cinchonin vom schwefelsauren Salz desselben ausging, fand ich nun die in Aether unlösliche Parthie als im wesentlichen aus Hydrocinchonin bestehend und das aus Aether und Benzin krystallisirende Alkaloïd, wie zu erwarten, als identisch mit Isocinchonin (Cinchonigin), während ich die in 
Aether lösliche, in Benzin aber unlösliche Base für identisch mit jener halte, welche beim Erhitzen von oxalsaurem Cinchonicin mit Schwefelsäure entsteht. Durch das Vorkommen der letzteren Base unter diesen Umwandlungsproducten des Cinchonins würde angedeutet werden, dafs im vorliegenden Falle ein Theil des Cinchonins zunächst in Cinchonicin übergeführt werde.

Der ganze Verlauf dieser Reaction erinnert übrigens lebhaft an einen Versuch, der vor langer Zeit von $W$ inckler *) mitgetheilt wurde. Dieser Chemiker erhitzte nämlich, um Cinchoninsulfat darzustellen, Cinchonin aus Versehen mit viel Vitriolōl und erhielt dann in der Hauptsache aus der braunen Lösung ein amorphes Sulfat, aus welchem er das amorphe Cinchonin darstellte, das eine fast weifse, jedoch in Aether unlösliche Masse darstellte. Für mich unterliegt es nun keinem $\mathrm{Zweifel,} \mathrm{dafs} \mathrm{dieses} \mathrm{amorphe} \mathrm{Cinchonin} \mathrm{mit}$ der von Caventou und Girard in ihrem Versuche erhaltenen ätherlöslichen Parthie übereinstimmt und jene Angabe der Aetherunlöslichkeit auf einer ungenauen Beobachtung von Seiten Winckler's beruht. Nimmt man bei der bezüglichen Ausschüttelung der Basen nicht viel Aether, so kann man sich leicht täuschen und dieselben für ätherunlöslich halten. Dafs Winckler das Alkaloïd nur amorph erhielt, beruht lediglich darauf, dafs er es nicht weiter $z u$ reinigen vermochte.

Noch möchte ich hinzufügen, dafs, soweit mir erinnerlich ist, de Vry vor mehreren Jahren aus den "amorphen Chinaalkaloiden" eine amorphe Base abschied, welche nach links polarisirte. Vielleicht bestand dieses Alkaloìd, über dessen Reinheit man im Zweifel sein mufste, zum Theil ebenfalls aus Isocinchonin.

*) Repertorium für Pharmacie 98, 381. 
Da das neutrale Rhodanat des Isocinchonins unlöslich in kaltem Wasser ist, so dürfte seine Abscheidung aus den amorphen Chinaalkaloïden auf keine besonderen Schwierigkeiten stofsen, obgleich dessen Löslichkeit hier durch andere Substanzen nicht unerheblich gefördert wird. Bei derartigen Untersuchungen dürfte daher bislang eine Verwechselung desselben mit Conchininrhodanat hin und wieder stattgefunden haben.

Wie aus Vorstehendem ersichtlich, entsteht das Isocinchonin unter sebr verschiedenen Bedingungen, und es bietet sich hin und wieder die Gelegenheit, es bei der Verarbeitung der Chinarinden beobachten zu können.

Charakteristisch für dieses Alkaloïd ist zweifellos sein Verhalten zum polarisirten Licht, indem es im Gegensatz zu allen anderen zweisäurigen Chinaalkaloïden, welche bis jetzt in dieser Beziehung untersucht wurden, ein Drehungsvermögen zeigt, welches mit zunehmender Concentration der Lösung zunimmt, auf Zusatz von Säuren dagegen abnimmt. Diese Erscheinung läfst sich nur damit erklären, dafs die Richtung, in welcher das polarisirte Licht durch das gelöste Molekũl des Isocinchonins hindurch geht, scheinbar bei dem Uebergang des Cinchonins in Isocinchonin von rechts nach links gedreht wurde. Da aber aus den polarimetrischen Bestimmungen noch deutlich hervorgeht, dafs die Constitution des Cinchonins dabei keine Aenderung erlitt, so ist jenes nur mōglich, wenn das Cinchoninmolekül aus zwei in einem Punkte mit einander verbundenen Atomgruppen besteht, von denen die eine optisch activ, die andere inactiv ist. Die Drehung würde sich dann nicht auf das ganze Cinchoninmolekül erstrecken, sondern nur auf eine Gruppe desselben. 